\title{
Proposal of the hybrid solution to determining the selected fracture parameters for SEN(B) specimens dominated by plane strain
}

\author{
M. GRABA* \\ Faculty of Mechatronics and Mechanical Engineering, Kielce University of Technology, \\ 7 Tysiąclecia Państwa Polskiego Ave., 25-314 Kielce, Poland
}

\begin{abstract}
In the paper, new hybrid (numerical-analytical) methods to calculate the J-integral, the CTOD, and the load line displacement are presented. The proposed solutions are based on FEM calculations which were done for SEN(B) specimens dominated by plane strain condition. The paper includes the verification of the existing limit load solution for SEN(B) specimen with proposal of the new analytical formulae, which were used for building hybrid equations for determining three selected fracture mechanics parameters.
\end{abstract}

Key words: fracture mechanics, fracture parameters, limit load, finite element method, single edge notched specimen.

\section{Nomenclature}

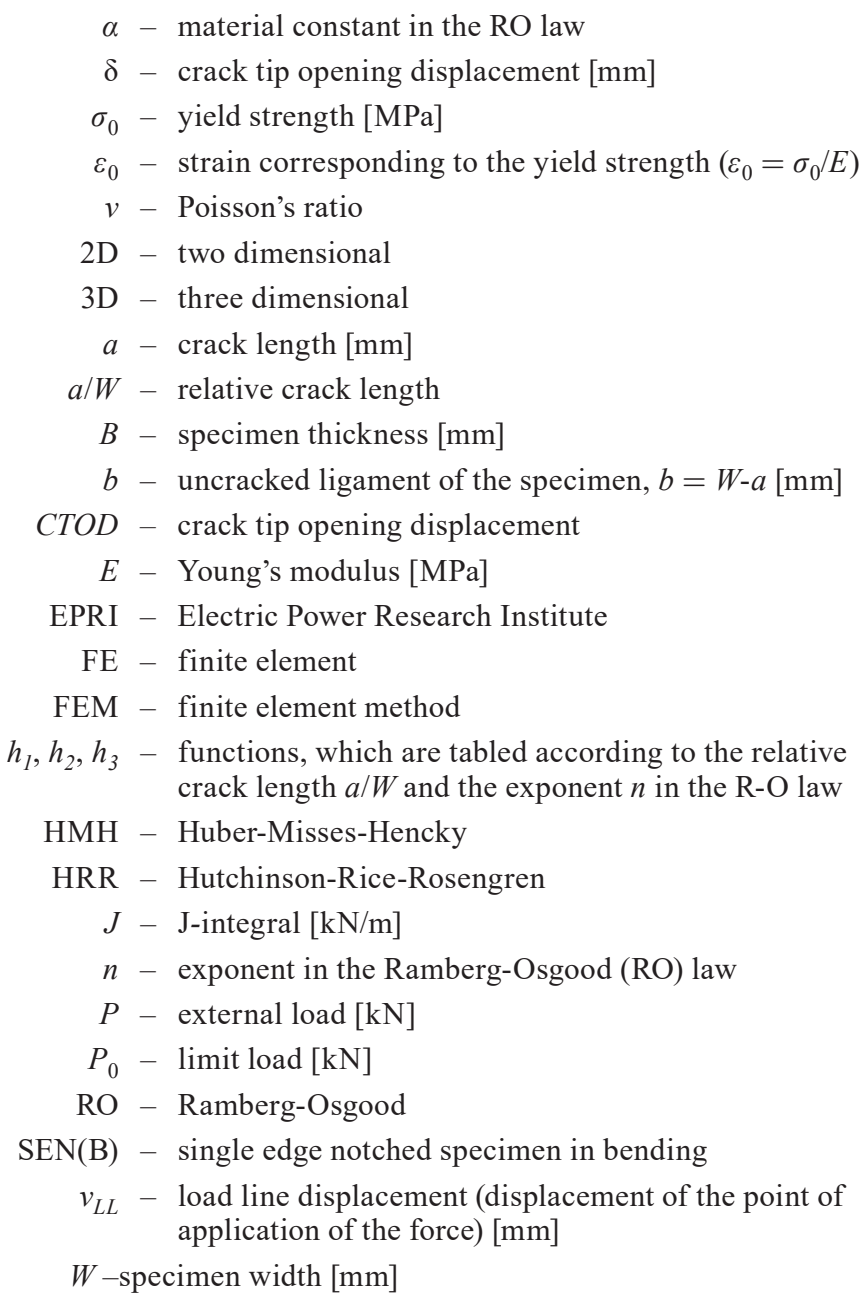

*e-mail: mgraba@tu.kielce.pl

Manuscript submitted 2016-10-11, revised 2016-12-09, initially accepted for publication 2017-02-07, published in August 2017.

\section{Introduction}

One of the basic approaches used in fracture mechanics is the Hutchinson, Rice, and Rosengren (HRR) solution first described in 1968 [1, 2]. For years this solution has been used to deal with elastic-plastic fracture mechanics problems which require determining the distribution of stresses around the crack tip, assessing fracture toughness or establishing the fracture criteria [3-7]. It is important to note that the solution has been modified many a time to provide new descriptions of stress fields [3]. The solution is not a perfect formula because it does not take account of the distribution of stresses, crack length, element (specimen) width, or thickness and type of external load (including the method of loading) [3]. Numerous attempts have been made to use the HRR formula $[1,2]$ to solve real engineering problems, as described in various papers and reports [8]. An important example is a document published in 1981, commonly known as the EPRI procedures $[3,9]$.

This well-prepared document provides the theoretical foundations of elastic-plastic fracture mechanics, as well as recommendations on how to use the knowledge of this discipline to solve practical engineering problems [3]. The EPRI procedures [9], which are based on a number of papers not to be described here, contain many practical suggestions on how to use fracture mechanics and how to determine the limit load for elements containing cracks. They also provide hybrid formulae to calculate the J-integral, the crack tip opening displacement (CTOD) denoted as $\delta$, and the displacement of the point of application of the force $v_{L L}$ [3]. It is important to note that when the recommendations given in the EPRI procedures [9] are followed, it is no longer necessary to conduct numerical calculations, which require developing an appropriate model complying with the applicable rules [3, 4, 10-12]. Since the EPRI procedures can be used to determine the value of the J-integral [9], they can be a practical engineering solution to analyse the strength of a structure with a crack, because the $\mathrm{J}$-integral is considered to be a pulling force of the crack and 
to control the distribution of stresses near the crack tip in elastic-plastic materials [3-4, 13].

To use the EPRI procedures [9], an engineer needs to know the geometry of the structure containing a crack and the material characteristics. They need to determine whether the plane strain or plane stress conditions are predominant and define the external load. The recommendations given in the EPRI procedures [9] refer to basic structural elements, i.e. plates, beams, or cracked cylinders. According to the SINTAP and FITNET procedures [13-15], all these structural elements can be used to idealise complex structures with a crack, in order to determine their fracture toughness and strength [3].

An interesting example is a single-edge notched specimen subjected to three-point bending SEN(B) (Fig. 1), tested for fracture toughness under plan strain conditions, according to the recommendations given in the standards [16-17]. When the recommendations [16-17] are followed and certain conditions are satisfied, the value of the J-integral for SEN(B) type specimens under predominant plane strain conditions can be treated as the material constant (denoted as $J_{I C}$ - elastic-plastic fracture toughness [16-17] ), and when converted into the critical value of the stress intensity factor $K_{I C}$, it can be used at the design stage according to the recommendations included in the procedures [14-15] and the Eurocodes [3, 18].

For the SEN(B) specimen, the authors of the EPRI procedures [9] propose the following set of hybrid formulae, which can be used in engineering for elastic-plastic analysis [3]:

$$
\begin{aligned}
& \begin{array}{l}
J=J_{e l}+J_{p l}=J_{e l}+\alpha \cdot \sigma_{0} \cdot \varepsilon_{0} \cdot b \cdot h_{1}(a / W, n) \cdot\left(P / P_{0}\right)^{n+1}, \\
\delta=\delta_{e l}+\delta_{p l}=\delta_{e l}+\alpha \cdot \varepsilon_{0} \cdot a \cdot h_{2}(a / W, n) \cdot\left(P / P_{0}\right)^{n}, \\
\mathrm{v}_{L L}=\mathrm{v}_{L L \_l}+\mathrm{v}_{L L \_p l}= \\
\quad=\mathrm{v}_{L L \_l}+\alpha \cdot \varepsilon_{0} \cdot a \cdot h_{3}(a / W, n) \cdot\left(P / P_{0}\right)^{n},
\end{array}
\end{aligned}
$$

where $J$ is the J-integral, $\delta$ is the crack tip opening displacement, $v_{L L}$ is the load line displacement, $\alpha$ is the material constant in the RO material, $\sigma_{0}$ is the yield stress, $\varepsilon_{0}$ is the strain corresponding to yield stress $\left(\varepsilon_{0}=\sigma_{0} / E\right)$, and $E$ is the Young modulus. In equations (1-3), subscripts " $e l$ " and " $p l$ " denote the "elastic part" and "plastic part" of the parameter, respectively. As shown in the EPRI procedures [2], the functions $h_{l}$, $h_{2}$, and $h_{3}$ are tabulated according to the relative crack length $a / W$ and the exponent $n$ in the RO law. In these formulae, the external load $P$ is normalised by the limit load $P_{0}$, which can be determined as [3]:

$$
P_{0}=0.728 \cdot \sigma_{0} \cdot b^{2} /(2 W),
$$

where $b$ is the length of the uncracked ligament calculated as $b=W-a$. The limit load should be interpreted as a state in which the whole uncracked ligament is plasticised. This suggests that the effective stresses calculated according to the $\mathrm{HMH}$ hypothesis are equal to or greater than the yield strength [3].

From formulae (1-3) it is clear that the basic assumption of the EPRI procedures [2] is to divide the parameters of elastic-plastic fracture mechanics into elastic $\left(J_{e l}, \delta_{e l}, v_{L L e l}\right)$ and plastic $\left(J_{p l}, \delta_{p l}, v_{L L_{-} p l}\right)$ parts. For the $\operatorname{SEN}(\mathrm{B})$ specimen, the elastic parts of the fracture parameters can be calculated as in [9] or as in Tada's handbook [19].

Formulae (1-3) seem simple to apply, but, as shown in [8], the results obtained with a finite element method (FEM) differ considerably from the data obtained according to the recommendations of the EPRI [3,9]. The same is observed for formulae used to determine the limit loads for other geometries, as described, for example, in $[3,20]$. Therefore, this paper proposes a new hybrid approach to calculate selected parameters of elastic-plastic fracture mechanics. The method is based on numerical and analytical calculations conducted by the author [3].
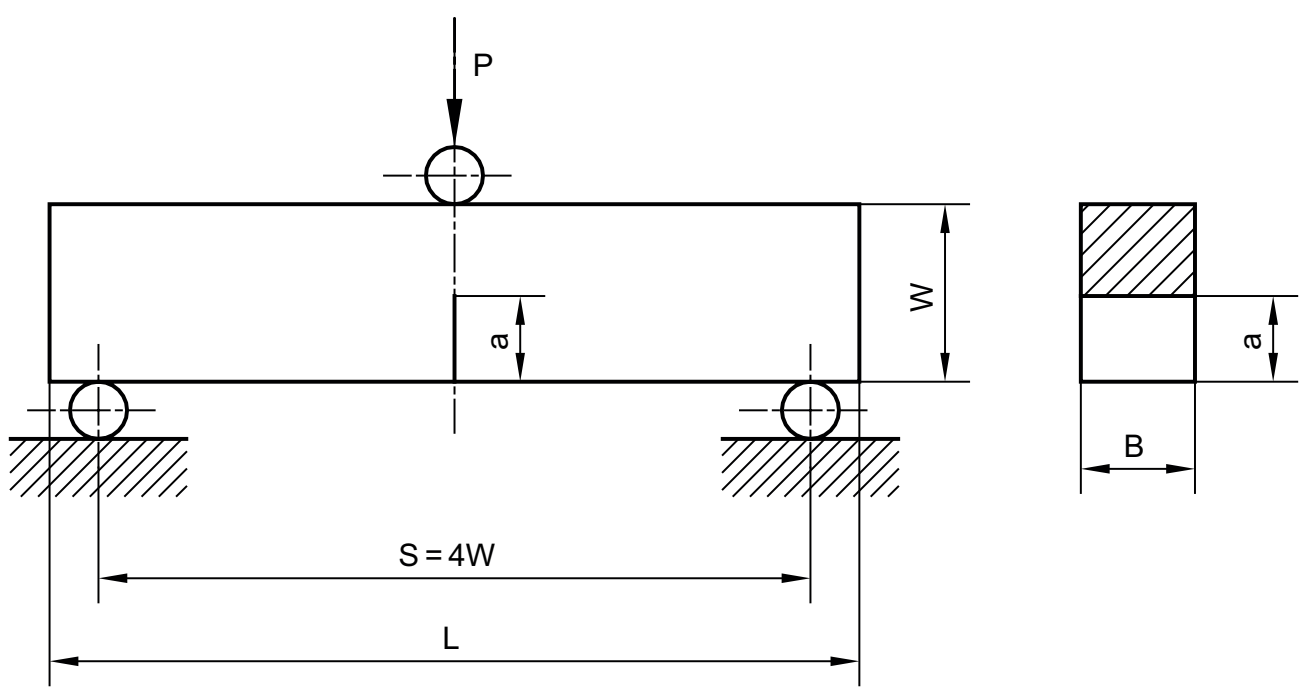

Fig. 1. Single-edge notched specimen subjected to three-point bending $\operatorname{SEN}(B)[3-4,8-9]$ 
Many scientific papers present discussion about the determination of CTOD, J-integral, or about the relationship between those parameters. In 1998, Shi et al. [21] were trying to determine the J-CTOD relationship for welded central cracked plates under tension. In 2012, Zhu and Joyce [22] verified many methods for determining the fracture toughness, including in their analysis the influence of the constrains [13]. Ruggieri and Paredes [23, 24] discussed the J-CTOD relationships for homogenous and welded single-edge notched plates under tension condition (SEN(T)). Based on numerical calculations, Ruggieri and Paredes [23, 24] proposed new empirical formulas for determining the J-integral and CTOD. A similar analysis for clamped SEN(T) specimens was presented in [25] - the authors proposed new formulas for calculating the J-integral and CTOD, which were based on three-dimensional numerical analysis. The influence of the geometrical constraints on CTOD was presented in [26], where the authors tried to summarize the relationships between CTOD and another elastic-plastic fracture parameters. All analyses presented in [26] were summarized using simple mathematical formulas. In 2014, Tagawa et al. [27], based on EPRI procedures [9], Shih concept [34], and numerical calculations for SEN(B) specimens, proposed equations similar to EPRI solutions, which may be used to determine the CTOD as a function of the external load. In [27], the authors presented empirical auxiliary equations, which are based on selected numerical results. The influence of the size of SEN(B) specimen on CTOD was discussed in [28]. In 2015, Sarzosa et al. [29] performed the analysis of the J-CTOD relationship for three-dimensional SEN(T) plates for both stationary and growing cracks. Also in 2015, Wang et al. [30] proposed empirical equations to calculate the J-integral and CTOD. In 2016, Antunes et al. [31] proposed the relationship between CTOD and external load for growing cracks under increasing fatigue loads.

As we can see, the J-CTOD relationship or dependence of the J-integral and CTOD of external load still lead to the formation of new scientific papers. This topic is still current, and the author of this paper will present a somewhat different approach to determining the J-integral or CTOD as a function of external load, which is an extension of results shown in [3].

\section{Details of numerical calculations}

The numerical calculations were performed using ADINA SYSTEM 8.8 [3, 32, 33]. The FE analysis involved verifying the limit loads for SEN(B) specimens with predominant plane strain conditions and solving elastic-plastic problems, for calculating the elastic-plastic fracture mechanics parameters [3]. A model of an elastic-perfectly plastic material was employed in the FE analysis to calculate the limit loads. In the calculations, the values of Young's modulus and Poisson's ratio and the four values of the yield strength were assumed to be constant $\left(E=206 \mathrm{GPa}, v=0.3\right.$, and $\sigma_{0}=\{315,500,1000,1500\} \mathrm{MPa}$, respectively) [3]. Four groups of materials differing in strength were considered. However, for calculating the elastic-plastic fracture mechanics parameters, the FE analysis was performed using a homogeneous isotropic model of an elastic-plastic material with the HMH plasticity condition, described by the following relationship [3]:

$$
\frac{\varepsilon}{\varepsilon_{0}}=\left\{\begin{array}{ll}
\sigma / \sigma_{0} & \text { for } \sigma \leq \sigma_{0} \\
\alpha\left(\sigma / \sigma_{0}\right)^{n} & \text { for } \sigma>\sigma_{0}
\end{array} .\right.
$$

The calculations were conducted assuming that the constant was $\alpha=1$ and that the four values of the exponent in the RO law were $n=\{3.36,5,10,20\}$ [3]. That gave a combination of 16 stress-strain curves, which can be classified according to the mechanical properties of ferritic steels, general-purpose structural steels, and materials exhibiting strong and weak strain hardening [3].

The calculations verifying the limit loads and the calculations in the elastic-plastic region were performed using the same FE model of the SEN(B) specimen. The numerical model was based on the recommendations provided in [3, 4, 10-12]. The existing axis of symmetry was used to model only half of the specimen. A contact problem was solved to represent the real behaviour of the specimen [3].

The crack tip of the SEN(B) specimen was modelled as a quarter of an arc with the radius $r_{w}$ ranging $1 \div 5 \mu \mathrm{m}$ [3]. This means that, in extreme cases, the radius of the crack tip was 40000 and 8000 times smaller than the specimen width $W$ [3]. The crack tip was divided into 12 elements. The density of the elements closer to the edges was higher; depending on the model, the edge elements were $5 \div 20$ times smaller than the largest elements located in the central part of the arc [3]. The size of the radius of the arc was conditioned by the level of the external load, as well as the crack length. For each specimen, the area near the crack tip with a radius of about $1.0 \div 5.0 \mathrm{~mm}$ was divided into $36 \div 50$ FEs, the smallest of which, located closest to the crack tip, was $20 \div 50$ times smaller than the element located furthest from the crack tip [3]. This means that, in extreme cases, the smallest element, located very close to the crack tip, constituted about $1 / 3024$ or $1 / 10202$ of the specimen width $W$, while the largest element modelling the area near the crack accounted for about $1 / 151$ or $1 / 240$ of the specimen width [3]. The parameters of the numerical model were strictly dependent on the crack length, material characteristics, and external load [3]. The analysis was conducted assuming small strains and small displacements [4] at the dominance of plane strain state. The FE model was developed using nine-node FEs of the 2-D SOLID plane strain type (mixed-order interpolation scheme) with nine integration points [3]. The total number of FEs used in the model was $6029 \div 6308$, exceeding the number of nodes, which ranged $24625 \div 25743$ [3].

In the numerical analysis, it was assumed that the width of the $\operatorname{SEN}(\mathrm{B})$ specimen was constant $(W=40 \mathrm{~mm})$, the distance between the supports was $S=4 \times W=160 \mathrm{~mm}$, the total length was $L=176 \mathrm{~mm}$, and the four relative crack lengths were $a / W=\{0.05,0.20,0.50,0.70\}$ [3]. It should be noted that the verification of the limit loads involved using 16 specimens, differing in the yield strength and the relative crack length. In the elastic-plastic analysis, however, 64 specimens were used. 
a)

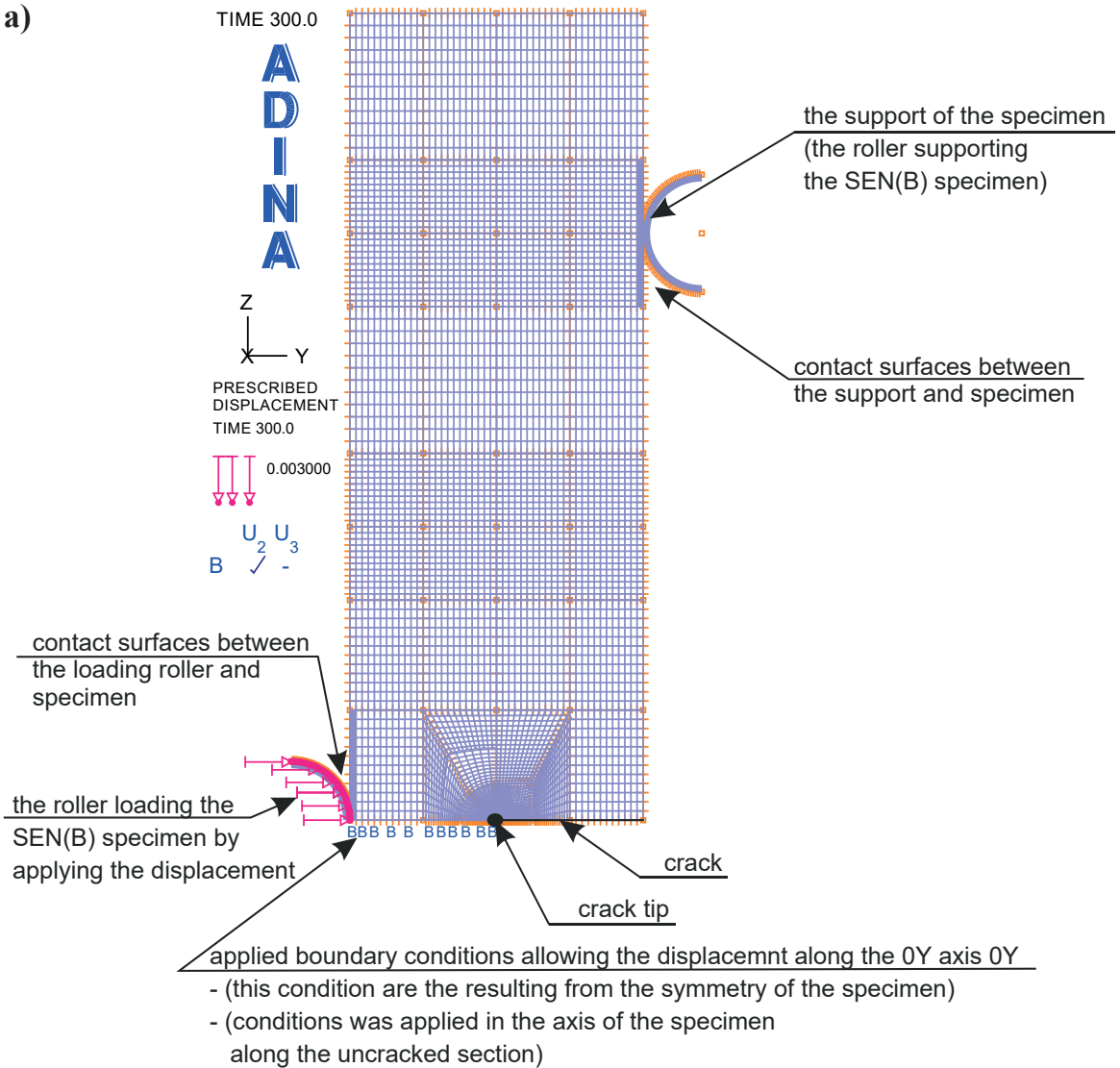

b)

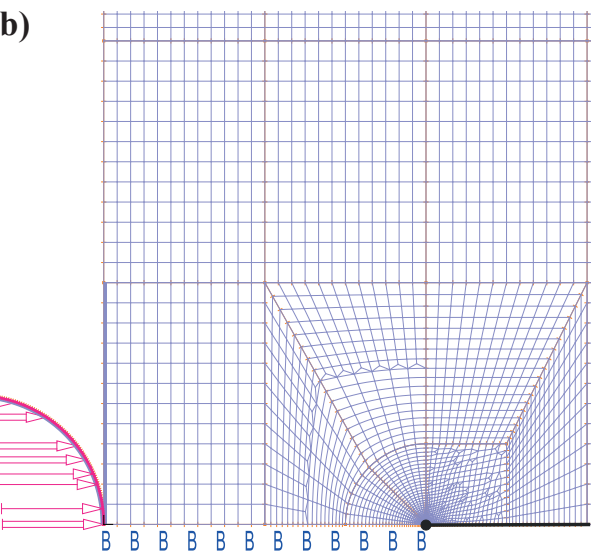

c)

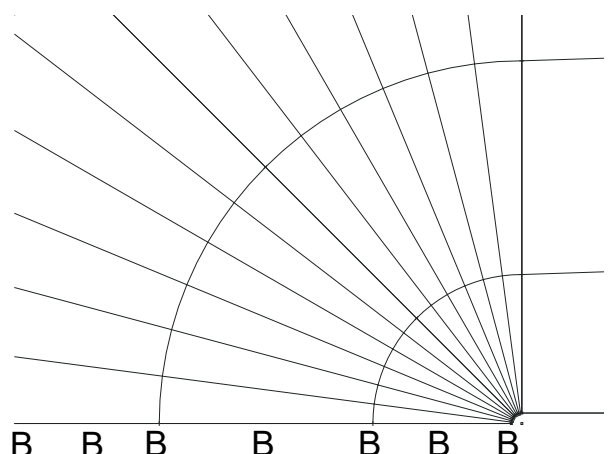

Fig. 2. Numerical model of the SEN(B) specimen under study: a) full model of the half of the SEN(B) specimen used in numerical analysis; b) larger fragment of the SEN(B) specimen - area near crack tip; c) the crack tip and the FE mesh in close proximity of the crack tip [3]

That required different definitions of the material and different crack lengths. Fig. 2 shows a numerical model of the SEN(B) specimen used in the FE calculations.

The elastic-plastic analysis was based on the plots showing the displacement of the point of application of the force $v_{L L}$, the J-integral, and the CTOD versus the external load P, normalised by the limit load $P_{0}$ [3]. The J-integral was determined

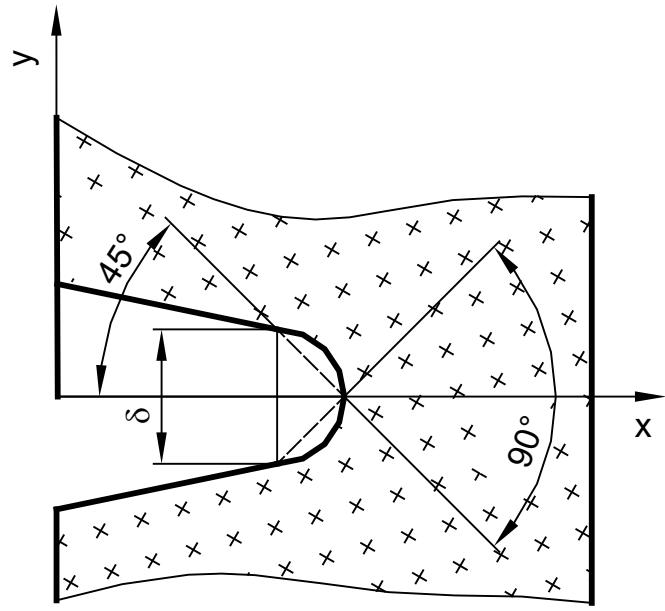

Fig. 3. Shih's concept [23] employed to determine the CTOD using the virtual shift method $[32,33]$ based on the concept of a virtual crack growth, to calculate the virtual change in energy $[3,32,33]$. The analysis used eight integration contours lying in the area comprised of all the finite elements. The radius of the finite elements located around the crack tip had a length of $\{10,15,20,25,30,35,40,45\}$ FEs. Each integration contour was generated in accordance with the recommendations given in $[4,10-12]$. It is important to note that the values of the J-integral obtained from the eight integration contours were similar. The CTOD was determined after the elastic-plastic FE calculations were completed using the concept proposed by Shih [34], illustrated in Fig. 3 [3].

\section{Results of numerical calculations}

3.1. Limit loads and their approximation. In the numerical analysis, the limit loads $P_{0}$ were determined by estimating the size and shape of the plastic zone increasing with external load and by analysing the diagrams showing the relationship between the force $P$ and the displacement of the point of application of the force $v_{L L}$. The limit load $P_{0}$ was assumed to be equal to the external load $P$ causing full plasticity of the uncracked ligament. The value of the load generally indicates the beginning of the saturation region in the $P=f\left(v_{L L}\right)$ curves. The numerical 
Table 1

Numerical values of the limit loads $\mathrm{P}_{0}$ and the percentage difference between the numerical solution and formula (5)

\begin{tabular}{|c|c|c|c|c|c|c|c|c|c|}
\hline \multirow{2}{*}{$\sigma_{0}[\mathrm{MPa}]$} & \multirow{2}{*}{$\sigma_{0} / E$} & \multicolumn{4}{|c|}{$P_{0_{-} F E M}[\mathrm{MN}]$} & \multicolumn{4}{|c|}{$\left(P_{0_{-} F E M}-P_{0_{-} E P R I}\right) / P_{0_{-} F E M} \cdot 100 \%$} \\
\cline { 3 - 10 } & & $a / W=0.05$ & $a / W=0.20$ & $a / W=0.50$ & $a / W=0.70$ & $a / W=0.05$ & $a / W=0.02$ & $a / W=0.50$ & $a / W=0.70$ \\
\hline 315 & 0.000153 & 3.983 & 2.952 & 1.172 & 0.432 & $3.922 \%$ & $-0.553 \%$ & $-2.209 \%$ & $-4.522 \%$ \\
\hline 500 & 0.000243 & 6.357 & 4.713 & 1.854 & 0.686 & $3.346 \%$ & $-1.150 \%$ & $-1.809 \%$ & $-4.549 \%$ \\
\hline 1000 & 0.000485 & 12.915 & 9.436 & 3.727 & 1.378 & $1.742 \%$ & $-1.251 \%$ & $-2.331 \%$ & $-4.877 \%$ \\
\hline 1500 & 0.000728 & 19.553 & 14.358 & 5.667 & 2.094 & $0.805 \%$ & $-2.650 \%$ & $-3.657 \%$ & $-6.142 \%$ \\
\hline
\end{tabular}

values of the limit loads and the percentage difference between the numerical solution and the formula recommended by the EPRI [9] - equation (5) - are provided in Table 1. Presented in the Table 1 numerical results were confirmed using the "twice elastic slope method", which may be used to determining the limit load base on the diagrams presenting external load versus load line displacement.

The analysis of the results concerning the values of the limit load leads to the following conclusions [3]. The longer the crack, the lower the value of the limit load [3]. The higher the yield strength, the proportionally greater the value of the limit load [3]. The numerical results are represented graphically in Fig. 4. The relationship between the limit load $P_{0}$ and the yield strength $\sigma_{0}$ is linear [3]. However, from Fig. 4 it is evident that for the relationship between $P_{0}$ and the relative crack length $a / W$, the linearity decreases with an increase in the crack length [3]. This phenomenon is likely to be affected by an increase in the size of the plastic zone at the interface between the specimen and the loading roller [3]. Similar observations were reported in $[35,36]$, which are concerned with the analysis of elastic-plastic stress fields.

As can be seen, the numerically calculated values of $P_{0}$ are only slightly different from those determined according to formula (5) [9]. For specimens with very short cracks, the values are up to $4 \%$ greater. However, as the crack length increases,

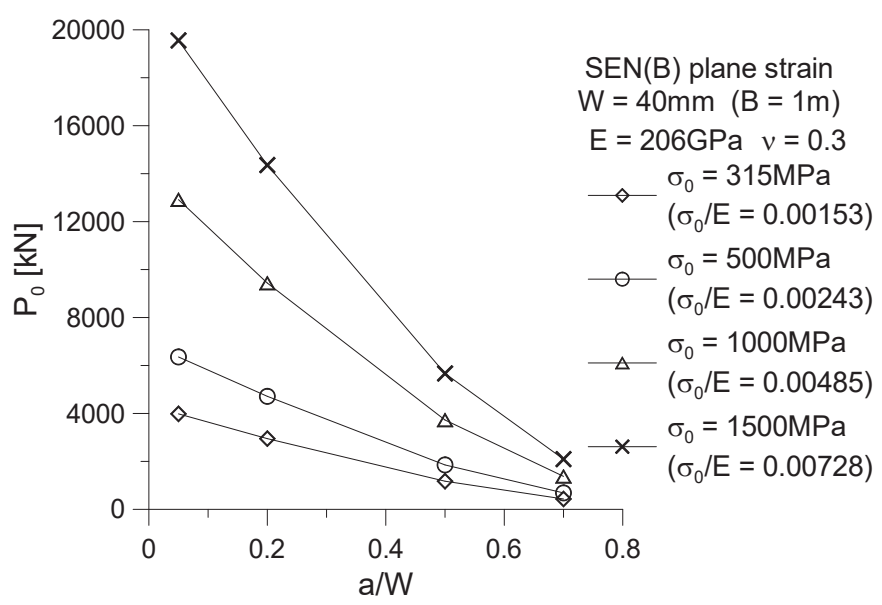

Fig. 4. Limit load $P_{0}$ versus relative crack length $a / W$ for the SEN(B) specimens with predominant plane strain conditions the difference between the FEM results and those obtained with formula (5) becomes greater (up to 6\%). It should be mentioned that the numerically calculated values are smaller than the values determined by means of formula (5) in accordance with the EPRI procedures [9].

The numerical results were then approximated. The algorithm presented in the EPRI procedures was used to write the formula for estimating the limit load $P_{0}$ for the $\mathrm{SEN}(\mathrm{B})$ specimens with predominant plane strain conditions [3]:

$$
P_{0}=B \cdot \sigma_{0} \cdot f(a / W)
$$

where the function $f(a / W)$ is given by:

$$
\begin{aligned}
f(a / W) & =0.01064 \cdot(a / W)^{3}-0.00032 \cdot(a / W)^{2}+ \\
& -0.02299 \cdot(a / W)+0.01398
\end{aligned}
$$

with $R^{2}=0.99963$ [3], or by:

$$
f(a / W)=[0.11958-0.11642 \cdot(a / W)]^{2},
$$

with $R^{2}=0.9991$. The use of equation (6) requires knowledge of the relative crack length $a / W$, the yield strength $\sigma_{0}$ expressed in [MPa], and thickness $B$ expressed in [m] [3]. The results of the limit load will be expressed in [MN] [3].

The alternative formula for calculating the limit load may be the empirical equation (8), describing the dependence $P_{0}=f\left(a / W, \sigma_{0}\right)$ in the following form:

$$
\begin{aligned}
P_{0} & =A_{1}+A_{2} \cdot \sigma_{0}+A_{3} \cdot(a / W)+A_{4} \cdot\left(\sigma_{0}\right)^{2}+ \\
& +A_{5} \cdot(a / W)^{2}+A_{6} \cdot \sigma_{0} \cdot(a / W),
\end{aligned}
$$

where the approximation coefficients are: $A_{1}=681, A_{2}=13.28$, $A_{3}=-7235, A_{4}=0.00017, A_{5}=10005$, and $A_{6}=-18.20$ for $R^{2}=0.998$.

\subsection{Analysis of the parameters of elastic-plastic fracture} mechanics and their approximation. The parameters of elastic-plastic fracture mechanics discussed in this paper are the J-integral, the CTOD, denoted as $\delta$ (which can be used to determine the actual fracture toughness $K_{I C}$ in accordance with the FITNET procedures [13-15], where the J-integral is addition- 
ally a parameter controlling the stress field near the crack tip $[4,5])$ and the displacement of the point of application of the force $v_{L L}$ [3]. The objective of the analysis was to determine the relationships between these parameters and the material characteristics (expressed by the strain hardening exponent $n$ and the yield strength $\sigma_{0}$ ) and the geometry of the $\operatorname{SEN}(\mathrm{B})$ specimens, expressed by the relative crack length $a / W$ (Fig. 5) [3].

The natural conclusion is that each of the three parameters increases with an increase in the external load (in the plots, normalised by the limit load $P_{0}$ ), while the rate of changes is conditioned by the material characteristics and the relative crack length [3]. The shorter the crack length, the higher the values of the J-integral, the CTOD $\delta$, and the displacement of the point of application of the force $v_{L L}$, with the values of the external load being constant (Fig. 5) [3]. Another finding is that the smaller the degree of material strain hardening (the higher the value of the exponent $n$ in the RO law), the higher the values of the parameters of elastic-plastic fracture mechanics, with the values of the external load being constant (Figs. 5a and 5f) [3]. An increase in the material strength (expressed by the yield strength $\sigma_{0}$ ) is accompanied also by an increase in the value of the J-integral, CTOD, and displacement of the point of application of the force $v_{L L}$, with the values of the external load being constant (Figs. 5d, 5e) [3].
The full graphical representation of the numerical results obtained in the FE elastic-plastic analysis is comprised of 192 curves [3]. As it is impossible to include them all either in this or any other journal paper, the numerical data (i.e. the values of the J-integral, the CTOD, and the displacement of the point of application of the force $v_{L L}$ as a function of the normalised external load $P / P_{0}$ ) was approximated.

In this way, a catalogue of hybrid - combined analytical and numerical - solutions can be created to determine these parameters with no need for FE calculations, as suggested by the authors of the EPRI procedures [3,9]. They applied an analytical and hybrid method to determine the parameters of elastic-plastic fracture mechanics, which involved estimating the parameters, separately as elastic and plastic (formulae (2-8)) [9]. The J-integral is determined experimentally in a similar way, i.e. separately as an elastic parameter and a plastic parameter, using a method based on changes in compliance with the ASTM standards [17]. In this study, the solution was simplified: the parameters of elastic-plastic fracture mechanics (the J-integral, the CTOD and the displacement of the point of application of the force $v_{L L}$ ) were not calculated separately as elastic parameters and plastic parameters. When the J-integral is determined in accordance with the Polish standard [16], using the single specimen method and the compliance calibration method, it is a)

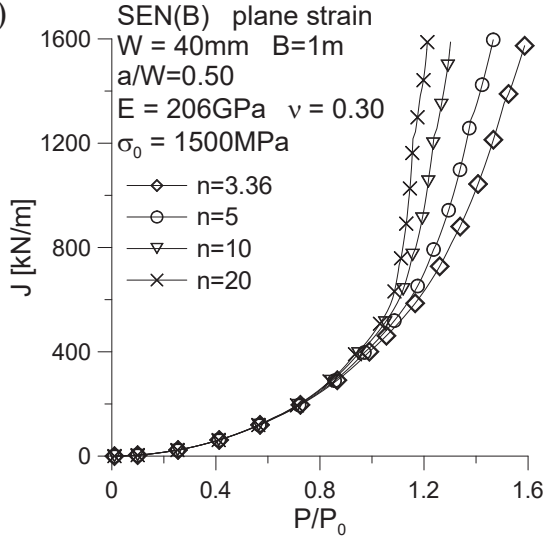

d)

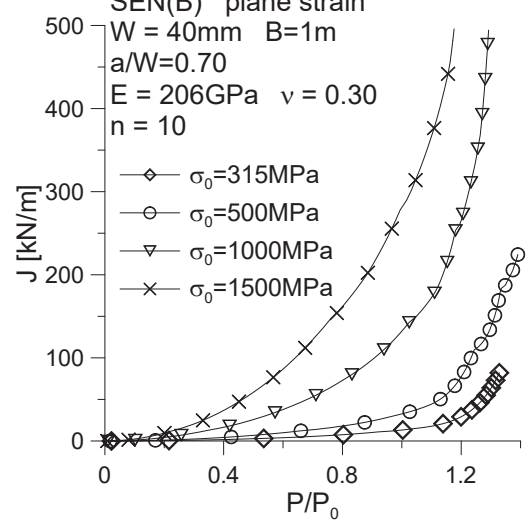

b)

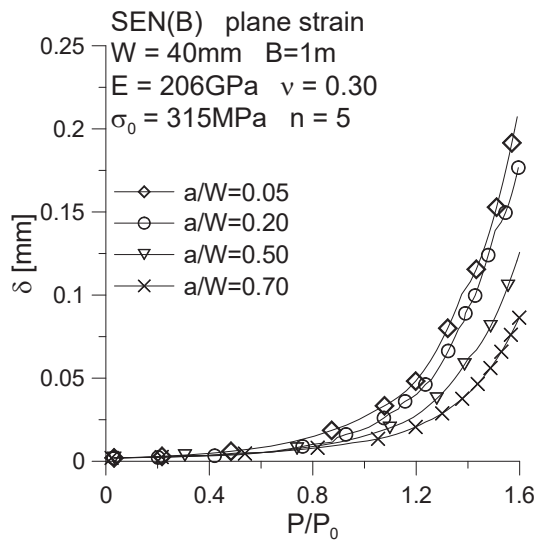

e)

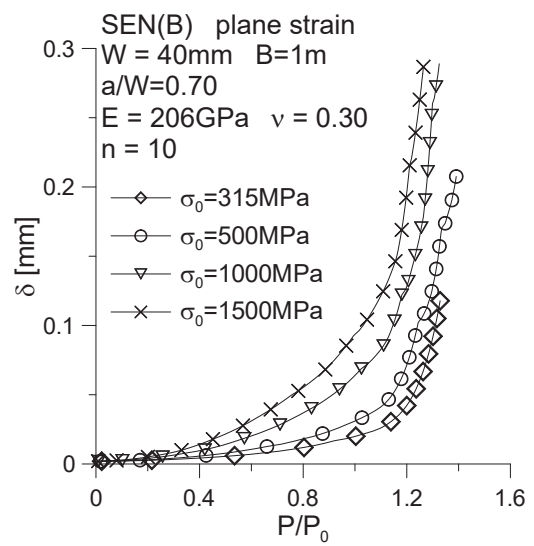

c)

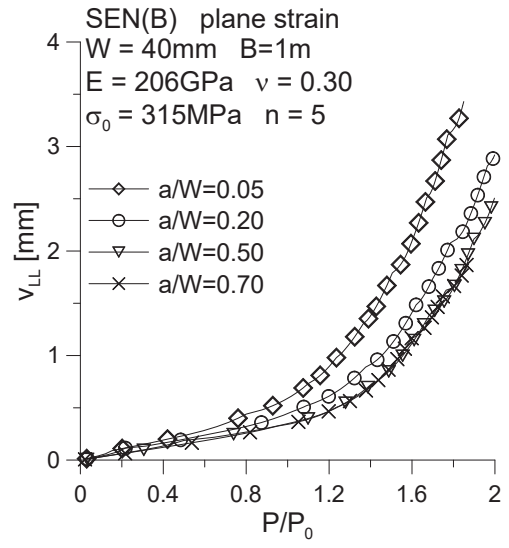

f)

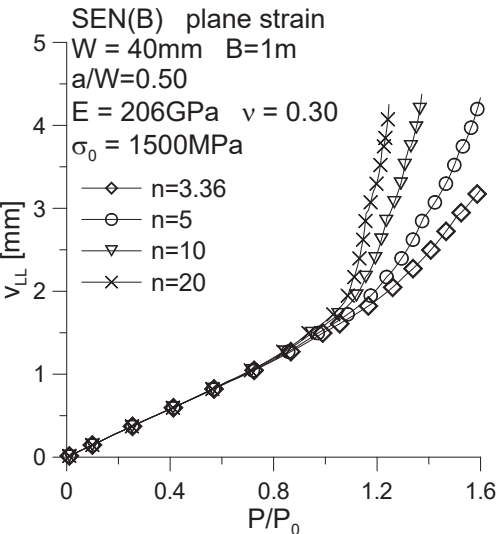

Fig. 5. The influence of: a) the exponent $n$ in the RO law and d) the yield strength $\sigma_{0}$ on the J-integral determined numerically; b) the relative crack length $a / W$ and e) the yield strength $\sigma_{0}$ on the CTOD, denoted as $\delta$, determined numerically; c) the relative crack length $a / W$ and f) the yield strength $\sigma_{0}$ [3] on the load line displacement $v_{L L}$; as the function of the external load $P$ normalized by limit load $P_{0}$ 
not necessary to perform separate calculations for the elastic and plastic parts. Therefore, it can be suggested that the J-integral, the CTOD and the load line displacement $v_{L L}$ should be determined as follows [3]:

$$
\begin{gathered}
J=\alpha \cdot \sigma_{0} \cdot \varepsilon_{0} \cdot b \cdot\left(P / P_{0}\right)^{n+1} \cdot\left[h_{1}^{*}(a / W, n)\right]^{n+1}, \\
\delta=\alpha \cdot \varepsilon_{0} \cdot b \cdot\left(P / P_{0}\right)^{n} \cdot\left[h_{2}^{*}(a / W, n)\right]^{n}, \\
\mathrm{~V}_{L L}=\alpha \cdot \varepsilon_{0} \cdot b \cdot\left(P / P_{0}\right)^{n} \cdot\left[h_{3}^{*}(a / W, n)\right]^{n},
\end{gathered}
$$

where $P_{0}$ is the limit load, calculated numerically for the purpose of this paper (Table 1, or formulae (6-7)), while the functions $h_{1}{ }^{*}, h_{2}{ }^{*}$, and $h_{3}{ }^{*}$ are dependent on the strain hardening exponent $n$ and the relative crack length $a / W[3]$.

The analysis of the results reveals that the values of the functions $h_{1}{ }^{*}, h_{2}{ }^{*}$, and $h_{3}^{*}$ are strongly dependent on the degree of material strain hardening expressed by the value of the strain hardening exponent $n$, the relative crack length $a / W$, and also the value of the external load $P / P_{0}$ (Figs. 6b, 6c). However, when the limit load $P_{0}$ is exceeded, the functions $h_{1}{ }^{*}, h_{2}{ }^{*}$, and $h_{3}{ }^{*}$ decrease towards the saturation region and reach almost a constant value (Fig. 6) [3]. The analytical and numerical method provided in the EPRI procedures [9], using the values of the J-integral, the CTOD, and the displacement of the point of application of the force, is based on constant values of the functions $h_{1}{ }^{*}, h_{2}^{*}$, and $h_{3}^{*}$ [9], which are independent of the external load [3]. Earlier studies by the author, described in [8], indicate that the values of the functions $h_{1}{ }^{*}, h_{2}{ }^{*}$, and $h_{3}{ }^{*}$ change with a change in the external load [3].

It is interesting that the functions $h_{1}{ }^{*}, h_{2}{ }^{*}$, and $h_{3}{ }^{*}$ are independent of the yield strength (Fig. 6). The values of the functions $h_{1}{ }^{*}, h_{2}{ }^{*}$, and $h_{3}{ }^{*}$ decrease with an increase in the external load $P / P_{0}$ until they reach the value of saturation with the external load being $P / P_{0} \geq 1.0$ (Fig. 6) [3]. All three functions increase with an increase in the crack length (for example, Fig. 6b). When the strain hardening exponent $n$ affects the value of the functions $h_{1}{ }^{*}, h_{2}{ }^{*}$, and $h_{3}{ }^{*}$ each of them needs to be considered separately. Initially, the value of the function $h_{1}^{*}$ increases with a decrease in the material strain hardening. However, after the external load is exceeded $\left(P / P_{0}=1.0\right)$, a slight shift in the relationship is observed. For $P / P_{0}>1.0$, the value of the function $h_{1}{ }^{*}$ rises slightly with an increase in the material strain hardening. The value of the function $h_{2}{ }^{*}$ rises over the whole range of external loads with a decrease in the degree of the material strain hardening (an increase in the exponent $n$ ). However, when the limit load is exceeded, the differences between the curves $h_{2}{ }^{*}=f\left(P / P_{0}\right)$ for the subsequent values of the strain hardening exponent are relatively small. The value of the function $h_{3}{ }^{*}$ increases with an increasing strain hardening exponent $n$, while the differences between the curves $h_{3}{ }^{*}=f\left(P / P_{0}\right)$ for the subsequent values of the strain hardening exponent $n$ increase with an increasing external load (Fig. 6c). These conclusions were drawn in the analysis of additional charts, not presented in the paper.

The results obtained in this study can be applied to solve real engineering problems, but representing them graphically may be troublesome. For this reason, it was essential to approximate the curves showing the relationship between the functions $h_{1}{ }^{*}, h_{2}{ }^{*}$, and $h_{3}{ }^{*}$ and the external load $P / P_{0}$. Since the functions $h_{1}{ }^{*}, h_{2}{ }^{*}$, and $h_{3}{ }^{*}$ are not affected by the yield strength and the functions change with increasing ratio $P / P_{0}$, the functions $h_{1}^{*}$, $h_{2}{ }^{*}$, and $h_{3}{ }^{*}$ were described using the following approximation equations [3]:

$$
\begin{gathered}
h_{1}^{*}(a / W, n)=F_{I}\left(P / P_{0}\right) / F_{I I}\left(P / P_{0}\right), \\
h_{2}^{*}(a / W, n)=a_{2}+b_{2} \cdot\left(P / P_{0}\right)^{-1}+c_{2} \cdot\left(P / P_{0}\right)^{-2}+ \\
+d_{2} \cdot\left(P / P_{0}\right)^{-3}+e_{2} \cdot\left(P / P_{0}\right)^{-4}+f_{2} \cdot\left(P / P_{0}\right)^{-5}+ \\
+g_{2} \cdot\left(P / P_{0}\right)^{-6}+h_{2} \cdot\left(P / P_{0}\right)^{-7}, \\
h_{3}^{*}(a / W, n)=F_{I I I}\left(P / P_{0}\right) / F_{I V}\left(P / P_{0}\right),
\end{gathered}
$$

a)

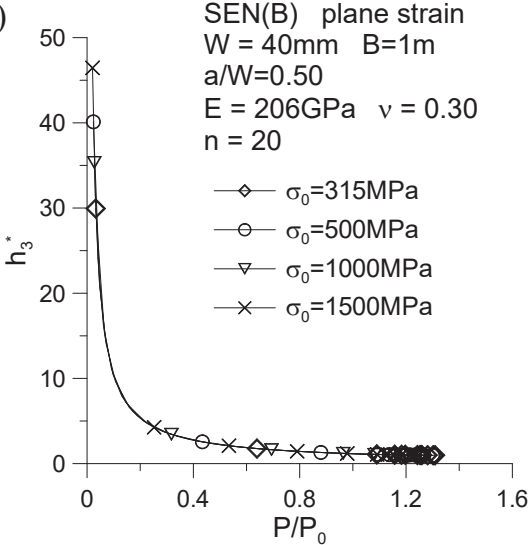

b)

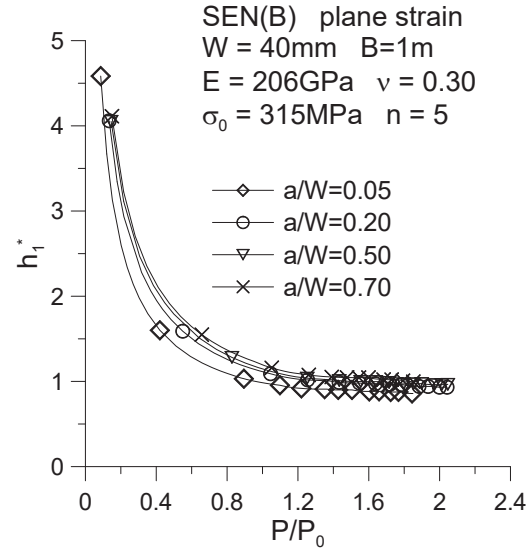

c)

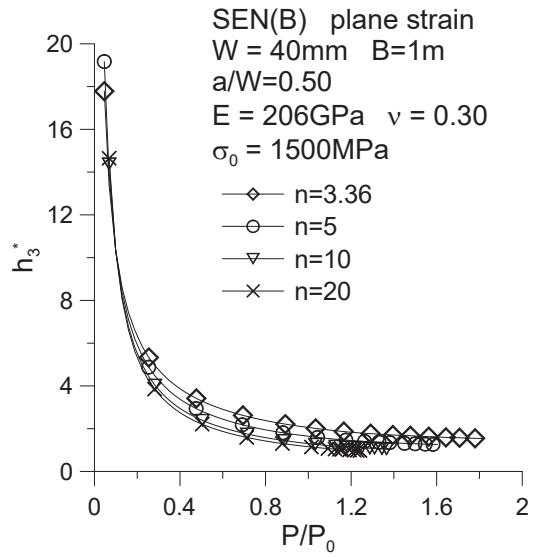

Fig. 6. Functions $h_{1}{ }^{*}, h_{2}{ }^{*}$, and $h_{3}{ }^{*}$ versus external load for the SEN(B) specimens, dependent on the material characteristics and the relative crack length - selected trajectories: a) $h_{3}^{*}$ versus $P / P_{0}$ for different yield strength $\sigma_{0}$; b) $h_{1}^{*}$ versus $P / P_{0}$ for different relative crack length $a / W$;

c) $h_{3}{ }^{*}$ versus $P / P_{0}$ for different strain hardening exponent $n$ 
wherein

$$
\begin{gathered}
F_{I}\left(P / P_{0}\right)=a_{1}+c_{1} \cdot\left(P / P_{0}\right)+e_{1} \cdot\left(P / P_{0}\right)^{2}+ \\
+g_{1} \cdot\left(P / P_{0}\right)^{3}+i_{1} \cdot\left(P / P_{0}\right)^{4} \\
\begin{aligned}
F_{I I}\left(P / P_{0}\right)= & 1+b_{1} \cdot\left(P / P_{0}\right)+d_{1} \cdot\left(P / P_{0}\right)^{2}+ \\
+ & f_{1} \cdot\left(P / P_{0}\right)^{3}+h_{1} \cdot\left(P / P_{0}\right)^{4}+j_{1} \cdot\left(P / P_{0}\right)^{5}, \\
F_{I I I}\left(P / P_{0}\right) & =a_{3}+c_{3} \cdot\left(P / P_{0}\right)+e_{3} \cdot\left(P / P_{0}\right)^{2}+ \\
& +g_{3} \cdot\left(P / P_{0}\right)^{3}+i_{3} \cdot\left(P / P_{0}\right)^{4}, \\
F_{I V}\left(P / P_{0}\right)= & 1+b_{3} \cdot\left(P / P_{0}\right)+d_{3} \cdot\left(P / P_{0}\right)^{2}+ \\
+ & f_{3} \cdot\left(P / P_{0}\right)^{3}+h_{3} \cdot\left(P / P_{0}\right)^{4}+j_{3} \cdot\left(P / P_{0}\right)^{5},
\end{aligned}
\end{gathered}
$$

where the approximation coefficients $a_{1} . . j_{1}, a_{2} . . h_{2}$, and $a_{3} . . j_{3}$ are dependent on the strain hardening exponent $n$ and the relative crack length $a / W$. Selected values of the approximation coefficients are shown in Tables 2-4. In the near future, the author intends to develop a computer application to assess the values of the functions $h_{1}^{*}, h_{2}^{*}$, and $h_{3}^{*}$, and, in consequence, the values of the J-integral, the CTOD, and the displacement of the point of application of the force $v_{L L}$, by introducing appropriate material characteristics and specimen geometry [3]. Currently, the best solution to take advantage of the proposed approximation, is to prepare a spreadsheet using the Microsoft Excel program.

Table 2

Approximation coefficients $a_{1} . . j_{1}$ required to calculate the values of the function $h_{1}{ }^{*}$ according to formula (12) - selected results

\begin{tabular}{|c|c|c|c|c|}
\hline \multicolumn{5}{|c|}{$a / W=0.05$} \\
\hline$n$ & 3.36 & 5 & 10 & 20 \\
\hline$a_{1}$ & 36.6 & 103.4 & 450.7 & 1345.2 \\
\hline$b_{1}$ & 402.4 & 595.5 & 1178.6 & 2215.7 \\
\hline$c_{1}$ & 2391.0 & 5637.9 & 19530.9 & 45703.4 \\
\hline$d_{1}$ & 7660.2 & 12618.3 & 29702.7 & 55644.0 \\
\hline$e_{1}$ & 11627.7 & 16936.5 & 27154.6 & 902.2 \\
\hline$f_{1}$ & 10503.5 & 9511.1 & 8302.8 & -25251.3 \\
\hline$g_{1}$ & -1788.1 & -14005.0 & -54569.9 & -83780.2 \\
\hline$h_{1}$ & -6893.3 & -14939.4 & -46211.9 & -63271.8 \\
\hline$i_{1}$ & -56.7 & 2943.3 & 19245.7 & 43918.1 \\
\hline$j_{1}$ & 1188.0 & 4018.9 & 18704.3 & 38626.3 \\
\hline$R^{2}$ & 0.9999 & 0.9999 & 0.9999 & 0.9999 \\
\hline
\end{tabular}

A comprehensive list of the coefficients of the approximation may be obtained by directly contacting the author of the paper, in the form of a text file, a Microsoft Excel file, or in
Table 3

Approximation coefficients $a_{2} . . h_{2}$ required to calculate the values of the function $h_{2}{ }^{*}$ according to formula (13) - selected results

\begin{tabular}{|c|c|c|c|c|}
\hline \multicolumn{5}{|c|}{$a / W=0.20$} \\
\hline$n$ & 3.36 & 5 & 10 & 20 \\
\hline$a_{2}$ & 0.72389 & 0.5562 & 0.3655 & 0.2009 \\
\hline$b_{2}$ & 0.0187 & 0.4236 & 0.6916 & 0.8490 \\
\hline$c_{2}$ & 0.0570 & -0.0062 & -0.0098 & -0.0079 \\
\hline$d_{2}$ & -0.0048 & 0.0006 & 0.0007 & 0.0005 \\
\hline$e_{2}$ & 0.0002 & $-1.95 \mathrm{E}-05$ & $-1.97 \mathrm{E}-05$ & $-1.37 \mathrm{E}-05$ \\
\hline$f_{2}$ & $-3.70 \mathrm{E}-06$ & $2.35 \mathrm{E}-07$ & $2.25 \mathrm{E}-07$ & $1.53 \mathrm{E}-07$ \\
\hline$g_{2}$ & $3.42 \mathrm{E}-08$ & $-9.56 \mathrm{E}-10$ & $-8.90 \mathrm{E}-10$ & $-5.99 \mathrm{E}-10$ \\
\hline$h_{2}$ & $-1.18 \mathrm{E}-10$ & 0 & 0 & 0 \\
\hline$R^{2}$ & 0,9877 & 0,9946 & 0,9988 & 0,9997 \\
\hline
\end{tabular}

Table 4

Approximation coefficients $a_{3} . . j_{3}$ required to calculate the values of the function $h_{3}^{*}$ according to formula (14) - selected results

\begin{tabular}{|c|c|c|c|c|}
\hline \multicolumn{5}{|c|}{$a / W=0.70$} \\
\hline$n$ & 3.36 & 5 & 10 & 20 \\
\hline$a_{3}$ & 392.3 & 767.2 & 1946.2 & 4554.4 \\
\hline$b_{3}$ & 770.5 & 1215.8 & 2443.6 & 5113.8 \\
\hline$c_{3}$ & 23634.8 & 40523.3 & 81875.6 & 181512.8 \\
\hline$d_{3}$ & 20201.7 & 36129.6 & 75971.5 & 174782.3 \\
\hline$e_{3}$ & 80790.5 & 96364.5 & 79167.2 & 90964.1 \\
\hline$f_{3}$ & 19980.5 & 29544.3 & 22628.9 & 28725.1 \\
\hline$g_{3}$ & -63902.8 & -127424.3 & -233763.9 & -524785.9 \\
\hline$h_{3}$ & -22531.5 & -58573.7 & -146892.3 & -410901.5 \\
\hline$i_{3}$ & 17418.6 & 39848.6 & 99415.6 & 268953.3 \\
\hline$j_{3}$ & 6024.7 & 19411.1 & 67052.7 & 220480.0 \\
\hline$R^{2}$ & 1.0000 & 1.0000 & 1.0000 & 1.0000 \\
\hline
\end{tabular}

the form of a proprietary computer application that allows for the set parameters of material and geometry to calculate the J-integral, the CTOD, and the load line displacement $v_{L L}$.

\section{Conclusions}

This paper has discussed the influence of the material characteristics and the relative crack length on three fracture mechanics parameters: the J-integral, the CTOD, and the displacement of the point of application of the force $v_{L L}$, calculated for selected elastic-plastic material configurations [3]. The study conducted for standard SEN(B) type specimens with predominant plane 
strain conditions involved a numerical (FE) analysis of the parameters and verification of the existing solutions recommended in the EPRI procedures [9] to determine the limit loads [3]. New analytical formulae based on FE calculations were proposed [3]. The results can be used to create a catalogue of combined analytical and numerical solutions to determine the J-integral, the CTOD, and the displacement of the point of application of the force $v_{L L}$ in relation to the external load, the material characteristics, and the relative crack length for SEN(B) specimens with predominant plane strain conditions [3]. The hybrid formulae (9-14) and the new formula to determine the limit load $P_{0}$ (equations (6-7) or (8)) can be applied to solve engineering problems in the area of the strength of structures containing cracks [3].

The presented new hybrid formulas allow to estimate selected parameters of the elastic-plastic fracture mechanics with more or less accuracy in relation to the actual experimental data. Using the proposed hybrid solutions, engineers should be aware that the set values of the J-integral, CTOD of the load line displacement true for the case of a stationary crack, and the possible use of these solutions for the case of growing cracks, should be subject to discussion and broad considerations.

The presented paper may be treated as a supplement of the author's earlier papers $[3,8]$, which discussed the hybrid method for determining the fracture mechanics parameters. The proposed new relationships for calculating the limit loads complete the broad consideration of fracture mechanics in this field, however, they do not exhaust the subject, as shown in [20].

Acknowledgements. The research reported herein was supported by a grant from the Faculty of Mechatronics and Machine Design at Kielce University of Technology (project No. 01.0.09.00/2.01.01.01.0027 MNSP.MKTM.17.002).

\section{REFERENCES}

[1] J.W. Hutchinson, "Singular behaviour at the end of a tensile crack in a hardening material", Journal of the Mechanics and Physics of Solids 16 (1), 13-31 (1968).

[2] J.R. Rice and G.F. Rosengren, "Plane strain deformation near a crack tip in a power-law hardening material", Journal of the Mechanics and Physics of Solids 16 (1), 1-12 (1968).

[3] M. Graba, "The hybrid method for determination of elastic-plastic fracture mechanics parameters for SEN(B) specimens", 25th Anniversary International Conference on Metallurgy and Materials, (2016).

[4] M. Graba, Numerical Analysis of the Mechanical Fields Near the Crack Tip in the Elastic-Plastic Materials. 3D Problems, PhD dissertation, Faculty of Mechatronics and Machine Building, Kielce University of Technology, Kielce, 2009, (in Polish).

[5] N.P. O'Dowd and C.F. Shih, "Family of crack-tip fields characterized by a triaxiality parameter - I. structure of fields", J. Mech. Phys. Solids 39 (8), 989-1015 (1991).

[6] A. Neimitz, M. Graba, and J. Gałkiewicz, "An alternative formulation of the Ritchie-Knott-Rice local fracture criterion", Engineering Fracture Mechanics 74 (8), 1308-1322 (2007).

[7] M. Graba, "The proposal of three-parametric fracture criterion of brittle materials", Przeglad Mechaniczny 2/2012, 24-31 (2012), (in Polish).
[8] M. Graba, "Verification of hybrid solutions to estimate the J-integral according to the EPRI procedures for the selected geometry", Proc. XXIV Symposium on Fatigue and Fracture Mechanics, (2012), (in Polish).

[9] V. Kumar, M.D. German, and C.F. Shih, An Engineering Approach for Elastic-Plastic Fracture Analysis, Electric Power Research Institute, Palo Alto, CA, 1981.

[10] M. Graba and J. Gałkiewicz, "Influence of the crack tip model on results of the finite element method", Journal of Theoretical and Applied Mechanics 45 (2), 225-237 (2007).

[11] W. Brocks, A. Cornec, and I. Scheider, Computational Aspects of Nonlinear Fracture Mechanics, pp. 127-209, GKSS-Forschungszentrum, Geesthacht, 2003.

[12] W. Brocks and I. Scheider, Reliable J-Values. Numerical Aspects of the Path-Dependence of the J-integral in Incremental Plasticity, pp. 127-209, GKSS-Forschungszentrum, Geesthacht, 2003.

[13] A. Neimitz, I. Dzioba, M. Graba, and J. Okrajni, The Assessment of the Strength and Safety of the Operation High Temperature Components Containing Cracks, Kielce University of Technology Publishing House, Kielce, 2008, (in Polish).

[14] Structural Integrity Assessment Procedures for European Industry. Final Procedure, Brite-Euram Project No. BE95-1426, British Steel, Rotherham, 1999.

[15] FITNET: European Fitness-for-service Network, FITNET report contract No. G1RT-CT-2001-05071, eds. M. Kocak, S. Webster, J.J. Janosch, R.A. Ainsworth, and R.Koers, (2006).

[16] Test Method for Determining the Fracture Toughness - the Critical Value of J-Integral - $J_{I C}$, PN-88/H-04336, Polish Committee for Standardization, Metrology and Quality, 1988, (in Polish).

[17] Standard Test Method for Measurement of Fracture Toughness, ASTM E 1820-05, American Society for Testing and Materials, 2005.

[18] PN-EN 1993 Eurocode 3: Stell Construction, 1993, (in Polish).

[19] H. Tada, P.C. Paris, and G.R. Irwin, The Stress Analysis of Cracks Handbook, Del Research Corporation, Hellertown, PA, 1973.

[20] S. Chauhan, J. Chattopadhyay, and B.K. Dutta, "Limit load equations for miniature single edge notched tensile specimens", Transactions of the Indian Institute of Metals 69 (2), 641-646 (2016).

[21] Y. Shi, S. Sun, H. Murakawa, and Y. Ueda, "Finite element analysis on relationships between the J-integral and CTOD for stationary cracks in welded tensile specimens", International Journal of Pressure Vessels and Piping 75, 197-202 (1998).

[22] X.-K. Zhu, J.A. Joyce, "Review of fracture toughness (G, K, J, CTOD, CTOA) testing and standardization", Engineering Fracture Mechanics 85, 1-46 (2012).

[23] C. Ruggieri, "Further results in J and CTOD estimation procedures for $\mathrm{SE}(\mathrm{T})$, fracture specimens - Part I: Homogeneous materials", Engineering Fracture Mechanics 79, 245-265 (2012).

[24] M. Parades and C. Ruggieri, "Further results in J and CTOD estimation procedures for $\mathrm{SE}(\mathrm{T})$ fracture specimens - Part II: Weld centerline cracks", Engineering Fracture Mechanics 89, 24-39 (2012).

[25] Y. Huang and W. Zhou, "J-CTOD relationship for clamped SE(T) specimens based on three-dimensional finite element analyses", Engineering Fracture Mechanics 131, 643-655 (2014).

[26] K. Han, J. Shuai, X. Deng, L. Kong, X. Zhao, and M. Sutton, "The effect of constraint on CTOD fracture toughness of API X65 steel", Engineering Fracture Mechanics 124-125, 167-181 (2014). 
[27] T. Tagawa, et. al, "A new CTOD calculation formula, considering strain-hardening property", Procedia Materials Science 3 , 772-777 (2014).

[28] M. Palombo, S. Sandon, and M. De Marco, "An evaluation of size effect in CTOD-SENB fracture toughness tests", Procedia Materials Science 109, 55-64 (2015).

[29] D.F.B. Sarzosa, R.F. Souza, and C. Ruggieri, "J-CTOD relations in clamped $\mathrm{SE}(\mathrm{T})$ fracture specimens including 3-D stationary and growth analysis", Engineering Fracture Mechanics 147, 331-354 (2015).

[30] E. Wang, W. De Waele, and S. Hertelé, “A complementary $\eta_{\mathrm{pl}}$ approach in J and CTOD estimations for clamped SENT specimens", Engineering Fracture Mechanics 147, 36-54 (2015).

[31] F.V. Antunes, S.M. Rodrigues, R. Branco, and D. Camas, "A numerical analysis of CTOD in constant amplitude fatigue crack growth", Theoretical and Applied Fracture Mechanics 85, 45-55 (2016).
[32] "ADINA: User interface command reference manual", vol. 1, Report ARD 11-2, 2011

[33] ADINA: Theory and modeling guide, vol. 1, Report ARD 11-8, 2011.

[34] C.F. Shih, "Relationship between the J-integral and the crack opening displacement for stationary and extending cracks", Journal of the Mechanics and Physics of Solids 29, 305-329 (1981).

[35] Y.J. Chao, X.K. Zhu, Y. Kim, P.S. Lar, M.J. Pechersky, and M.J. Morgan, "Characterization of crack-tip field and constraint for bending specimens under large-scale yielding", International Journal of Fracture 127, 283-302 (2004).

[36] M. Graba, "The influence of material properties and crack length on the Q-stress value near the crack tip for elastic-plastic materials for centrally cracked plate in tension", Journal of Theoretical and Applied Mechanics 50 (1), 23-46 (2012). 